\title{
CONTROL SYSTEM MOBILE ROBOTS WITH MODUlaR ARCHitecture AS A MUlTi-Agent System WiTh A HIERARCHICAL TOPOLOGY
}

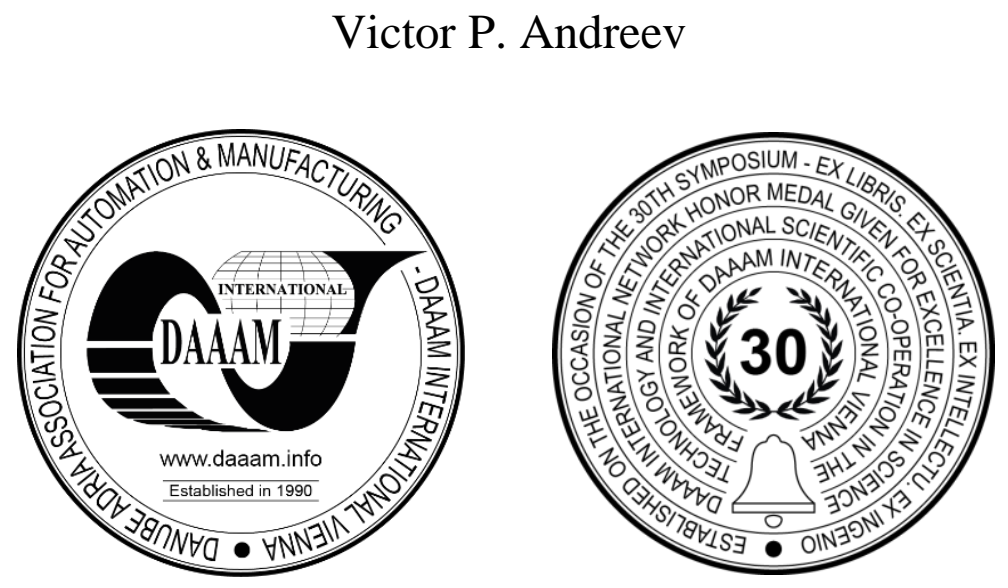

This Publication has to be referred as: Andreev, V[ictor] (2019). Control System Mobile Robots with Modular Architecture as a Multi-Agent System with a Hierarchical Topology, Proceedings of the 30th DAAAM International Symposium, pp.0010-0019, B. Katalinic (Ed.), Published by DAAAM International, ISBN 978-3-902734-22-8, ISSN 1726-9679, Vienna, Austria

DOI: $10.2507 / 30$ th.daaam.proceedings.002

\begin{abstract}
An approach to solving an urgent scientific problem is considered - the development of principles and methods for creating control systems of mobile robots with a modular architecture based on the theory of multi-agent systems. It is proposed to use the hierarchical topology of the robot control system. Modular architecture assumes that each mechatronic module has its own control system implemented on relatively cheap microcontrollers. The modular solution allows for the rapid reconfiguration of robotic systems and significantly reduce the cost of manufacturing robots by reducing their variety. Inter-modules information and control interaction is proposed to be organized on a hierarchical basis - a group of full-featured mechatronic submodules, solving the same type of tasks and working together, are controlled by a mechatronic module of a higher level. This approach allows increasing the system performance due to the distribution of computational load between computing devices of individual mechatronic modules and submodules. The proposed control system can be represented as a multi-agent system consisting of full-featured mechatronic and/or electronic modules - agents that interact with each other to achieve a specific goal. In this structure, an analogy is seen with such area of scientific research as a multi-agent systems.
\end{abstract}

Keywords: mobile robot; modular robot; distributed control system; multi-agent system; computer network.

\section{Introduction}

Most modern mobile robots (MR) have a monoblock design and a corresponding control system. In this design, the sensor and control system of the robot has a single center for the control and processing of sensory information using a computer with a sufficiently large computing power. This design has a number of disadvantages. First, the specificity of the work in progress performed determines the robot construct, which leads to the need to have a wide variety of robotic systems. Consequently, financial costs are growing for the production of a large number of various robots and the production of work. The second, the algorithmic complexity of the tasks of a robotic system control grows with an increase in the functional capabilities of MRs, as well as with the expansion of the conditions for their use. Consequently, the requirements for the computing power of the Central computer are constantly increasing, which increases the power 
consumption of the MR, its cost and weight-and-dimensional characteristics. Thirdly, the complexity and cost of the repair work of this equipment is increasing. The obvious way to solve these problems is to create robots with a modular architecture. However, there are new problems associated with the organization of coordinated inter-module interaction in such a distributed control system. At the same time, such a control system should have the property of reconfigurability without the software code redoing of both individual modules and the system as a whole. Therefore, it is necessary to search for methods for implementing the computational process of control and decision-making based on distributed computing performed on a multiple of processors. The solution of this problem will significantly reduce the computing power requirements of individual computers of a multiprocessor system and increase the performance of the system as a whole.

To date, there is no clear definition of the concept of "Robots with modular architecture." This research area may include both the developments of homogeneous [1-4], and heterogeneous [5-8] robots. Recently, a new area in this research has separated, which is rather a search for design methods aimed at providing developers and manufacturers of MR with universal modular architectures. The purpose of such architectures is to simplify the process of developing robotic and mechatronic devices using ready-made universal modules.

As an example, the system H-ROS [9], which is based on the framework ROS. This system allows one to build and control components that are compatible with H-ROS. The user only needs to program the robot components responsible for the cognition ("brain" of the robot) and independently develop program applications for specific tasks. In this case, the user does not need to solve the problem of integrating various technologies and interfaces. However, the use of $\mathrm{H}-$ ROS requires a special single-board computer on each component used to run ROS-2, which imposes certain restrictions on the use of this system.

Another example is the modular architecture of the miniature mobile platform AMiRo [10]. The proposed solution has a powerful hardware with small dimensions, open source software, distinct division of functions and tasks between modules, the ability to work with external applications and the ability to connect other devices (modules). However, reconfiguration due to the inclusion of third-party modules may be difficult due to the complex multi-level software architecture, as well as the small size of the robot.

An example of creating modular architectures by combining different modules (drives, sensors, controllers, etc.) with each other is the research [11]. Here, the authors propose a software framework R2P (Rapid Robot Prototyping), which is designed to create robots based on standardized hardware modules with the appropriate software and the developed Real-time CAN (RTCAN) interaction protocol. R2P provides easy integration of mostly simple modules, without taking into account more complex devices (for example, entire mobile platforms).

A similar modular architecture of RoboCAN is discussed in the article [12]. The architecture can be embedded in the Robotics Integrated Development Environment (RIDE) or other similar environments. Similar to R2P, the RoboCAN framework allows the integration of various actuators, sensors and control devices into mobile robot architectures (mostly simple components). The software of the modules is regardless of microcontrollers used; this allows one to create complex devices without modifying the existing approach in the implemented solution.

Analysis of works in the field of modular robot architectures shows that most of them offer some way of creating standard software and hardware interfaces between the modules to ensure the robots reconfigurability. In this case, as a rule, such a fundamental requirement for the design of the MR sensor and control system as the problem of providing real-time control under the condition of increasingly complicated MR functionality and the need to use relatively lowpower microprocessors or single-board computers (embedded computing devices) in the modules is not taken into account.

\section{Distributed mobile robot control system with modular architecture}

In the previously published work of the author [13] it is proposed to create a distributed control system for a modular MR. It is the modular architecture of the control system that allows one to divide the overall control task into subtasks according to the functional-modular principle, and distribute these subtasks among the full-featured robot modules. This implementation of distributed computing provides a significant reduction in the computational load on the computing device of each module, which allows the use embedded computing devices in the control system of individual modules.

The functional-modular architecture is based on the principle of full functionality of modules [14], which is formulated as follows: each robot module should be able to perform its goal function in any convenient way, using only its own means to execute commands from an external control system. This article discusses the principles and methods of constructing the software and hardware of the MR control system with a modular architecture. The proposed structure is quite simple and it can be automatically reorganized in the "plug-and-play" mode. 
Based on the principle of full functionality, it is proposed to consider the structure of the MR control system (in the minimum version) as a synergetic union of full-featured modules (fig.1) [15]: Transport Module - TM, Power Module PM, the sensory system, consisting of Short-Range Sensor Module - SRSM and Long Range Sensor Module - LRSM, modules of an impact on the environment - SPM (manipulators, machining process, grippers etc.) and Intelligent Control Module - ICM. In this structure, each module is responsible for only one function of a robotic system.

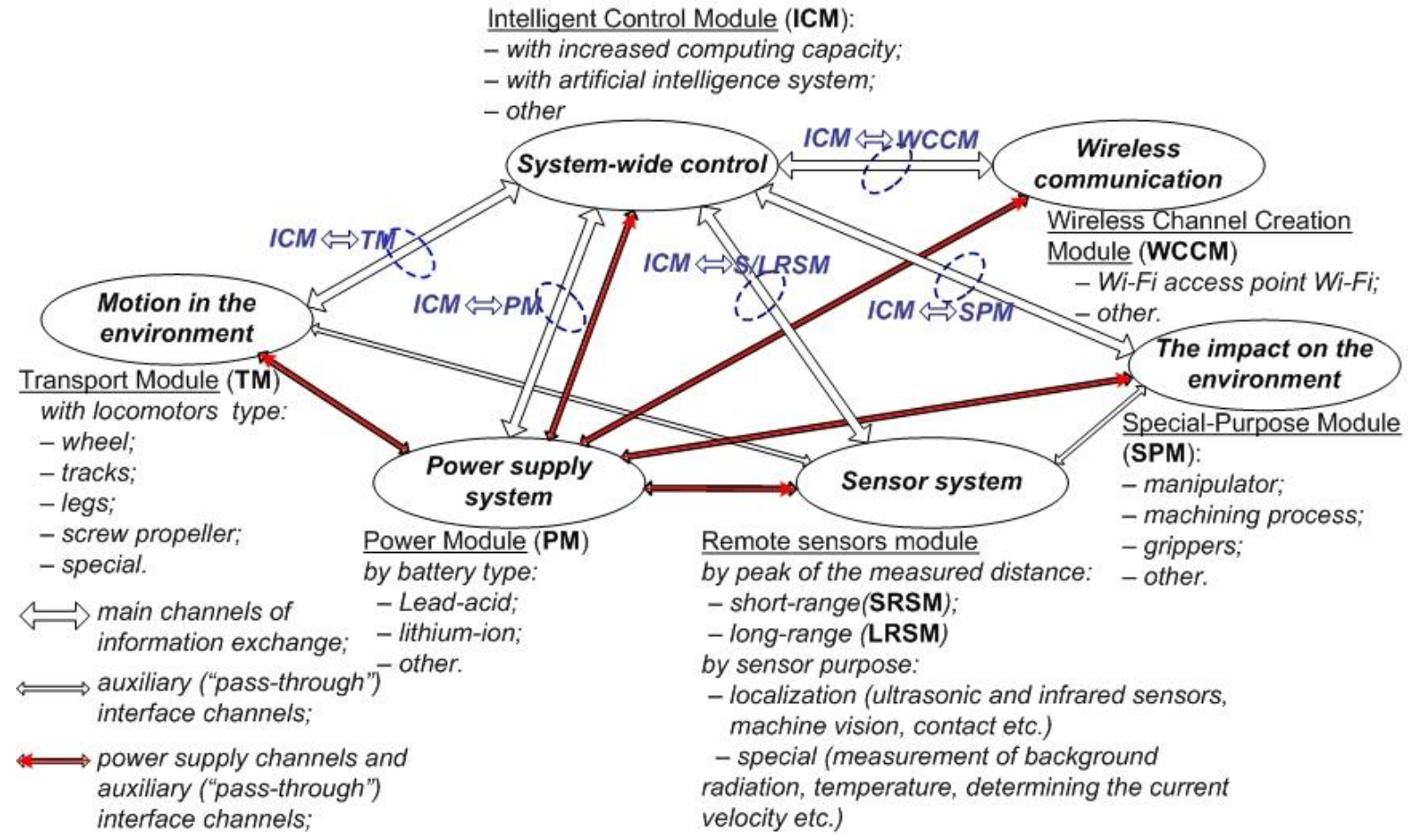

Fig. 1. Functional-modular architecture of a control system of a mobile robot

The system-wide control function is implemented in the Intelligent Control Module (ICM), which is a supervisor in relation to all other robot modules. The ICM does not manage the task execution process of this module. In our case, the ICM forms the control goal and controls only the result of its achievement by a Worker module. This functional completeness is the main difference between our approach and others, in which the module-supervisor controls the operation of all modules at the executive level.

The transport function is implemented by the transport module (TM). The task of the TM is to move the transport platform with the load (it may be a manipulator) from the current position to the location specified by the ICM module. The module can be equipped with different locomotors types: wheels, tracks, legs, screw propeller, etc. Naturally, the motion control algorithms for TM with different locomotors type will be different but they must be implemented by the module's own control system without redoing its the program code. This is its full functionality. If necessary, the TM control system can directly (bypassing the ICM) make a request to the MR sensor system, thereby reducing the load on the ICM processor and the data transmission channel. Therefore, it is necessary to have a direct interaction (in the figure 1 "pass-through" channel) between the TM and the Sensor system.

The information function is implemented by a remote Sensor system consisting of several sensor modules (SM): the short-range sensors module (SRSM) and the long-range sensors module (LRSM). The task of the modules is the detection of obstacles and objects of manipulation, as well as the determination of their geometric and dynamic parameters. These modules can, upon appropriate requests, provide sensory information to other modules directly (bypassing the ICM), or even give them a command to suspend their functions in special cases, for example, to prevent a possible collision of the MR with an obstacle. The ICM may prohibit the execution of this command if the collision is the necessary MR actions. The algorithm of direct information interaction between full-featured modules should take into account the presence in each module of its own sensor system, including sensors of the module internal state.

The technological function (and partially the transport function) is the environmental impact. The function is implemented by special purpose modules (SPM). These modules are manipulators-actuators (MAM) and the gripping modules (GM), which provide the ability to use different tools for moving manipulation objects within the robot workspace. The mechanical operations modules (MOM) also implement a technological function, since they are designed to perform various technological influences on manipulation objects - drilling, milling, etc.

The communication function is implemented by the network infrastructure of an information interaction between modules (indicated in Fig. 1 by multidirectional arrows). The MR communication with an external supervisor - the human operator of the robotic complex - is formed by a special Wireless Channel Creation Module (WCCM). Figure 1 shows 
the network topology of a star type, but a bus and mixed topology is possible. The choice of network topology is likely to depend on a number of factors that have yet to be determined.

The power function is implemented in the power module (PM). This module provides power to the electronic and electromechanical components of all robot modules. Its functions also include the safe switching on-off of the modules electronic components: the correct shutdown of microcontrollers, saving of the file system state, monitoring and displaying of the batteries state etc. Switching off the power supply to all modules (switching off the robot) is performed only by the ICM command. In this case, the PM performs a special modules shutdown algorithm, requesting their readiness for shutdown. The system should allow the shutdown of individual modules also at the command of the ICM in the case when they are not needed to perform work at the present moment (for example, to save energy).

In [13] the author proposed the structure of a distributed stabilized power supply. Its feature lies in the fact that the general unstabilized power supply of all robot modules, as well as power supply of electric drives, is carried out from a single battery, which is part of the PM. At the same time, each module is equipped with its own Unit for generating the required set of Stable Voltages (SVU). Voltage is supplied to the input SVU directly from the battery, but under the control of ICM. When the input voltage ripples caused, for example, by the starting currents of the electric motors, the presence of an intra-module SVU eliminates the possible interruption of the program code execution by the computing devices of the modules. Such an organization of power supply allows not imposing strict restrictions on the required number of stabilized voltages when developing a new module for the robot. In addition, in case of exceeding the total power consumption, it is enough to install additional batteries in the PM or replace the PM itself by installing a module with a larger battery capacity. In addition, the heat generated during voltage stabilization is distributed between the modules, and is not concentrated in a single power supply, which would require the installation of radiators. Since the PM has a wired network to modules provide power, it can be used both for organizing the information interaction of the PM with all modules, and as a communication channel for inter-module information interaction. It is clear that such a communication channel has a low bandwidth. Therefore, it can be used as a backup channel for transmitting commands, but not for transmitting a large information stream.

The given functional-modular architecture of the mobile robot control system reflects only the general approach. Subsequent experiments with laboratory models of modular MR showed the need for further development of this architecture. The results of such studies carried out under the RFBR Grant 16-07-00811a "Development of functionalmodular principle of software and hardware design of intelligent mobile robotic systems" are published in [13 - 20].

For experimental verification of the proposed methods and solutions the laboratory models of mobile robots with a modular architecture were developed and manufactured (Fig. 2), consisting of 4 variants of the transport module with different locomotors type (two wheel TM of different design, TM with omnidirectional wheels and walking TM), power module (PM), ultrasonic sensor module of parallel action (SRSM) and intelligent control module (ICM). Inter-module information interaction is implemented by combining the computing devices of the modules into a local area network.

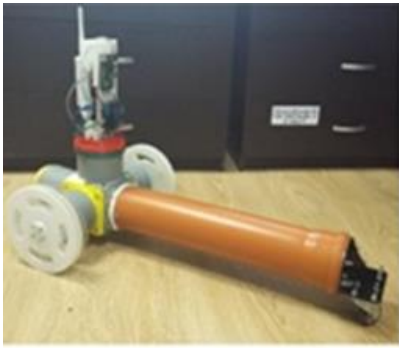

a)

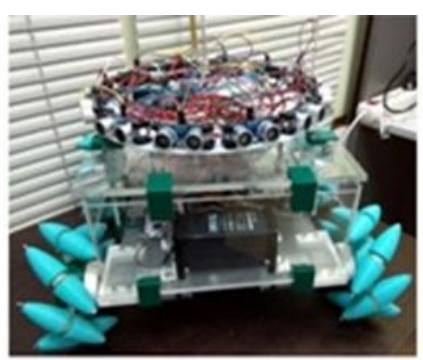

b)

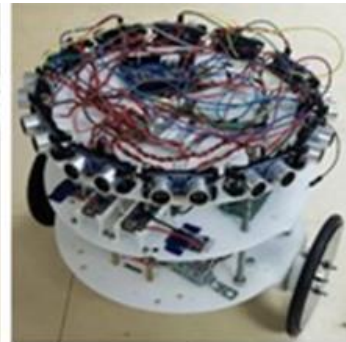

c)

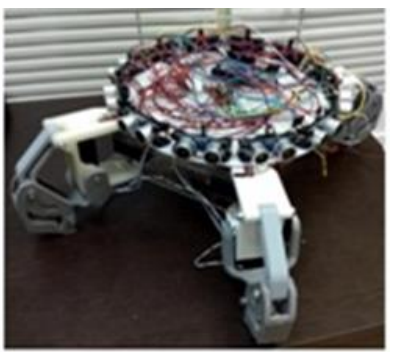

d)

Fig. 2. Laboratory models of mobile robots with modular architecture: $a$ - wheel robot (option 1), $b$ - omnidirectional robot, c - wheel robot (option 2), d - walking robot

The experiments showed that the proposed architecture with two-level hierarchy is not able to provide the implementation of the control process in real time by embedded computing devices, since the algorithms of the control system of functional modules are quite complex. The amount of processed information in the modules is still large, and the computing power of embedded devices does not provide a real-time mode. Particularly expensive are the process of sensory information analyzing and its fusion, as well as the implementation of decision-making algorithms at the level of a system-wide intelligent control module. It is necessary to find a fundamental solution to this problem, since as the tasks solved by MR both in stand-alone and in supervisor mode become more complicated, the algorithms of the module control system will become more complicated and the use of microcomputers with higher performance will still lead to the limited functionality of the module. 
Experiments with the TM showed that in order to solve the indicated problem, further development of the modular architecture of a MR is possible. The way of further development is to divide the full-function modules into submodules in order to create a multi-level hierarchical (pyramidal) network topology of the control system. This topology will reduce the algorithmic complexity of the computing process in submodules, which will allow the use of low-performance microprocessors. It is proposed to organize inter-module control interaction according to a hierarchical principle - a group of full-function mechatronic submodules that solve the same type of tasks and work together are controlled by a mechatronic module of a higher level. Inter-modular interaction in the system is implemented according to the "Master Worker group" principle. This approach will increase the system performance due to the implementation of distributed computing on computing devices of modules and submodules, as well as the implementation of the reconfiguration property is simplified. However, in this case, the role of inter-module interaction algorithms significantly increases.

We believe that the principles of creating such a pyramidal structure can be based on the theory of multi-agent systems, which will allow using the achievements in this field for the implementation of distributed computing in the MR control system.

\section{Hierarchical topology of the MR control system and multi-agent systems}

Multi-level hierarchical (pyramidal) topology assumes that the computational control process of a full-function module can be divided into subfunctions similarly to the method described in the previous section. Then, the implementation of these subfunctions can be assigned to the submodules (Workers), providing each submodule with its own control system built on a separate computing device. The control of such submodules is assigned to the top-level module (Master). At the same time, the full-function of the modular structure of the "Master-Workers group" type must be preserved. For example, the MR transport module consists of several actuator-submodules that are included in the TM control system. Each actuator-submodule can be executed also as a separate full-function submodule with its own a sensory and control system, but its functionality is simplified. The microprocessor of the actuator-submodule control system should be responsible only for the motor operation, the parameters of which are set by the TM control system (Master): the position of its output link, the speed vector, etc. Such submodule should report information about its current state both to other similar submodules in the group (Workers group) and to the supervisor (Master). Similarly the sensor modules (Workers group), for example, process the readings of groups of identical sensors, filter and convert a data into the required format for its transmission to the modules of next level hierarchy (Master), which perform the data fusion.

The upper level hierarchy modules are responsible for complex, behavioural tasks - coordination of the subordinate modules work: the mobile platform moves the robot to a given point in space and independently performs obstacle avoidance; the remote sensors module builds a distances map, etc. In the process of accomplishing the MR general task, inter-modular information interaction is dynamically performed, which ensures the coordinated operation of all modules and submodules.

Analysis of the construction principles of multi-agent systems showed that in our approach to the creation of a control system for robots with modular architecture, certain analogies are observed [20], whose application in the field of modular robotics has not yet been widely considered in well-known publications. The principles of dividing the general control process into subfunctions that do not violate the property of the full functionality of modules are not yet known. The optimal types of communication channel topologies at different levels of the hierarchical network structure have not been determined. Requirements to interfaces of inter-module information interaction are not formulated, the corresponding network protocols are not developed. The conditions and mechanisms for combining inter-modular intra-level and interlevel information interaction have not been developed at all. For hierarchical organization of the network structure of the control system, it is also necessary to develop requirements and conditions (including network protocols) that provide the ability to quickly reconfigure and scale the modular structure of the robot depending on its purpose. Methods for remote dynamic reprogramming of modules computing devices have not been developed. We believe that the analysis of the properties of multi-agent systems and the methods of organizing information interaction in such systems will help to find solutions to the considered problems.

"Intelligent multi-agent systems - one of the new promising areas of artificial intelligence, which was formed on the basis of research results in the field of distributed computer systems, network technologies for solving problems and parallel computing" [21]. Our approach, which consists in creating a hierarchical network structure of an MR control system, is fully correspond with the above definition of an intelligent multi-agent system. It has already become clear that the process of complex interactions of an autonomous robot with the environment in real time can be implemented only through distributed computing - no, even promising, mono-computer is not able to implement such an algorithm. Therefore, we believe that the solution of this problem is possible with the use of distributed computer systems. In the considered functional-modular architecture of the MR control system (see Fig.1) at its representation in the form of hierarchical structure an analogy with such areas of research in the information technology field as distributed information systems, computer networks, methods of artificial intelligence is clearly seen. Achievements in these areas are integrated in research, united by the common name "multi-agent systems". 


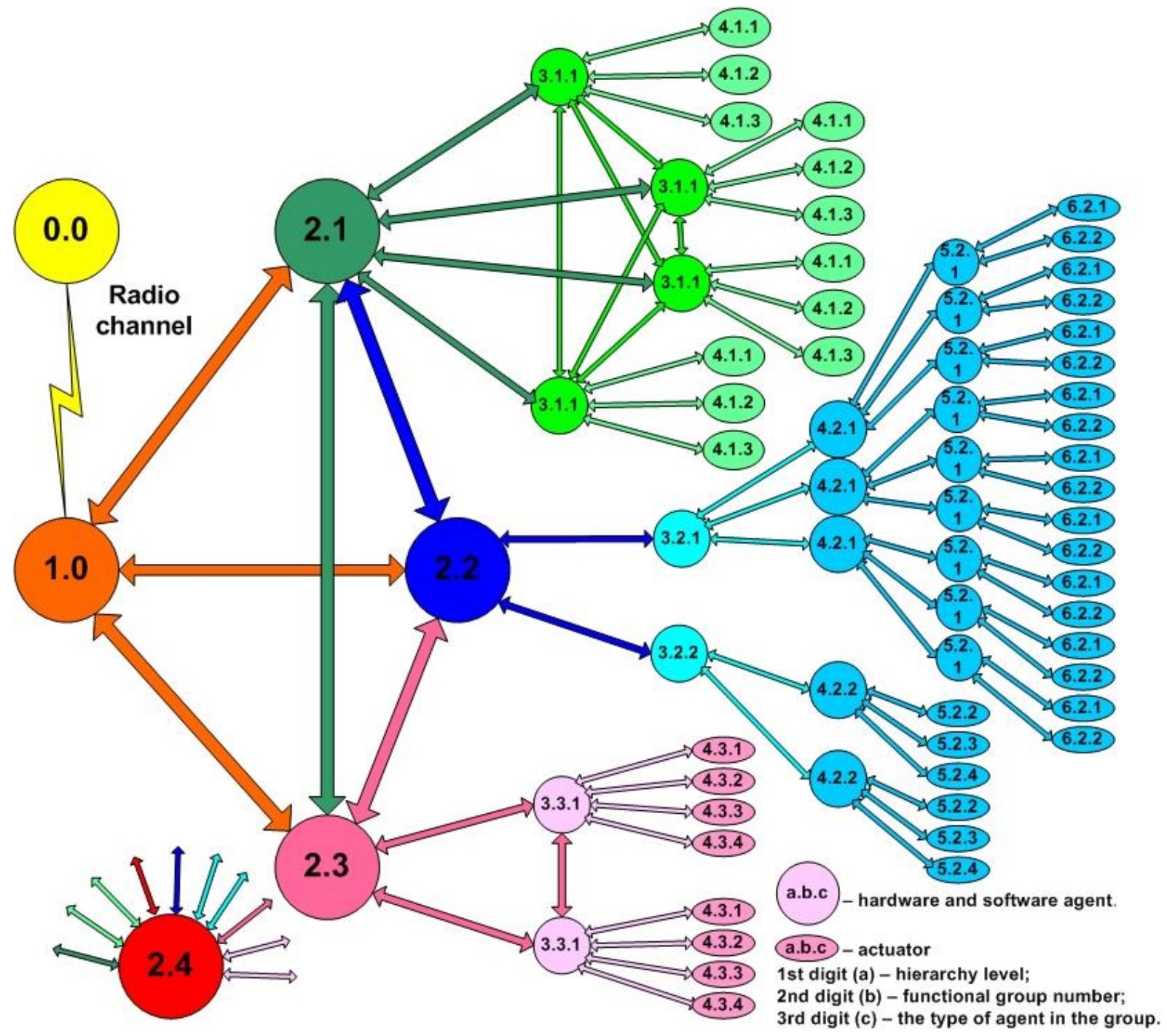

Fig. 3. Multi-agent system - information measuring and control system of mobile robot

Figure 3 shows one of the possible options for the implementation depicted in Fig.1 of the architecture of the MR control system in the form of a multi-agent system built on a hierarchical basis. Agent with an index of 0.0 is a MR remote control panel (person-operator of a mobile robot). This agent interacts with the agent with index 1.0 over the radio channel, which is the agent-module ICM (intelligent control module according to Fig.1) and is located at the first level of the hierarchy. The ICM agent, in accordance with its functional purpose, performs the formation of tasks for the agent-module TM (agent with index 2.1), agent-module SM (agent with index 2.2), agent-module SPM (agent with index 2.3) and agentmodule PM (agent with an index of 2.4), which are located at the 2nd level of the hierarchy. All agents of the second level of the hierarchy have communication channels with each other. Information interaction between modules of the same hierarchy level, if necessary, should be carried out directly, and not through the top-level module. This requirement reduces the computational load on this module and reduces traffic between hierarchy levels. Such an interaction, for example, between a TM and a SRSM will allow a transport module to move in a dynamic environment without participate in the control of the ICM agent, eliminating possible collisions with obstacles in relatively simple cases.

The third level of the hierarchy is formed from agents with index 3.1.1. These agents are software and hardware modules of a locomotors control systems: wheels, legs, etc. Agents with an index of 4.1.1, which are located at the fourth level of the hierarchy, are actuators equipped with appropriate sensors. All agents 3.1.1 have communication channels for interacting with each other, since during the TM movement, each agent needs to know the state of the other agents at every moment of time, especially in the case of moving on the rough terrain. The presence of horizontal communications violates the hierarchical principle, but it can be justified for a specific technical implementation of the interaction logic. The number of agents 3.3.1 can vary, for example, from 3 to 8, depending on the TM design: three-wheeled - 8-wheeled; 4-legs - 8-legs, the combination of legs-wheels, etc. In addition, the functionality of this agent can be changed - master (with actuator) or passive (sensors only). Agents with indexes 3.2.1 and 3.2.2 also belong to the third level of the hierarchy and, according to Fig.1, they are SRSM and LRSM. SRSM (with indices 3.2.1) is most rational to realize on ultrasonic distance sensors that can be located along the perimeter of the MR and create a range map. An example of such a sensor 
module is given in [19]. Due to the large computational complexity of simultaneously processing ultrasound signals from multiple ultrasound sensors (agents with an index of 6.2.1), this processing is parallelized by using a similar pyramidal architecture - agents of the 4th and 5th levels (with indices 4.2.1 and 5.2). The SRSM can also have a set of infrared (IR) distance sensors (elements with an index of 6.2.2), for example, duplicating the readings of ultrasonic sensors. However, the presence of six levels of hierarchy here may not be necessary - there may be more or less; it depends on the total number of sensors, the complexity of the algorithm for fusing their readings and microprocessor performance. Most likely, the functional of agents 3.2.1, 4.2.1 and 5.2.1 should be the same. In this case, inter-modular information interaction between agents of the same hierarchy level is absent. This is explained by a sequential, from level to level, information processing in the absence of the mutual influence of localized information; here the process of segmentation is performed - restoration of the areas connectedness. However, in some cases, such interaction may be necessary, for example, when implementing the lateral braking algorithm."

Agent with index 3.2.2, i.e. LRSM can be a stereoscopic vision system that performs the integration of video information from the left and right cameras (indices 5.2.2 - 5.2.4). Video data from 2 cameras, for example, from each of the 3 zones of the view field, are fused by agents with an index of 4.2.2. When connecting other remote sensors, this branch of the hierarchical tree can be expanded, for example, by connecting thermal imagers or laser scanners. It can be assumed that due to the huge flow of video information received at the input of vision system, the hierarchy tree will consist of a significantly larger number of levels than shown in the figure. However, here it may be necessary to limit the number of hierarchy levels due to time delays caused by the processing of information at each level.

An agent with index 2.3, which corresponds to the SPM in Fig. 1, performs the role of coordinator of 2 manipulators controlled by their own control systems, which are designated as two identical agents with index 3.3.1. These agents actually implement the multi-link manipulator control system (in Fig.3-three-link with agents-actuators 4.3.1 - 4.3.3), equipped with a gripper (agent-actuator 4.3.4). Here, similar to agents with index 3.1.1, an information interaction channel between agents 3.3.1 is required.

Agent with index 2.4, which corresponds to the PM (Fig.1), has information interaction with all modules and submodules of the hierarchy, since its function is to monitor and control the state of their power supply, as described earlier. The main purpose of this module is to provide power to all electronic systems of agents-modules. Therefore, this module must have direct electrical contact with each module and submodule. Then, the information communication of this module with the rest can be technically realized directly through the same wires as the power supply of the modules, bypassing the chain of agent-modules. The bandwidth of such a communication channel is extremely low, so this channel can be used as a backup communication channel with low frequency of information exchange and its small volume.

Inter-modular communication channels in this hierarchical structure of the MR control system can be constructed based on networks various topologies: "bus", "star", or a combination thereof. The use conditions of certain types of networks, topologies and network protocols come yet to be determined.

\section{Multi-agent systems properties and its analogies in the control system with the hierarchical topology}

In our hierarchical system, knowledge and resources are distributed among rather "independent" agents-modules. This follows from the principle of full functionality. The system also has a program module for general command control the base agent ("resident" in the terminology of multi-agent systems). As in multi-agent systems, scripts are generated and executed by each agent-module independently. Agents-modules using their sensory system respond to unpredictable events, and the reaction can be independent, or carried out in interaction with a resident [22] (for example, the human operator MR).

"An agent is an entity that resides in some environment from which it receives data and that reflects events occurring in the environment, interprets them, and executes commands that affect the environment. The agent can contain software and hardware components... The lack of a clear definition of the agents world and the presence of a large number of attributes associated with it, as well as the availability of a wide variety of agents examples suggests that agents are a fairly common technology that accumulates several different areas» [23]. In our case, the "agent" is not only a program; it is a hardware-software solution of a module control system that provides its full functionality.

In the agents' theory, there are two definitions of an intelligent agent - "weak" and "strong" [23]. Intellectual agent (IA) in a weak sense has the following properties [24]:

- autonomy - the ability of IA to function without human intervention and at the same time implement self-control over its actions and internal state;

- social ability - the ability to function in a community with other agents, exchanging messages with them using some generally understood communication language;

- reactivity - the ability to perceive the state of the environment and promptly respond to the changes that occur in it; 
- pro-activity - the ability of an agent to take the initiative, i.e. the ability to generate goals and act rationally to achieve them, and not only to respond to external events.

According to this definition, the agent-module in our structure has the property of autonomy; this follows from the previously formulated principle of the module's full functionality. In our modular architecture (see Fig. 1), each agentmodule necessarily has an information interaction channel with other agent-modules; therefore, it has the property of social ability. The only difference is that the interaction is not implemented on the principle of "everything with everyone"; interaction is organized according to a hierarchical principle, and between agents of the same hierarchy level.

In our structure, each agent-module is able to perceive the state of the environment and timely respond to the changes that occur in it. It is realized both by means of module internal state sensors, and by means of remote sensors of the sensor module with which there is an information interaction. Therefore, each agent-module has a reactivity property. However, in our case, not all agents-modules can / must have the property of pro-activity; one of the research objectives is to determine the conditions under which the agent-module can or should possess this property.

An intelligent agent in the strong sense is characterized by the presence of "at least some subset" of additional properties called "mental properties" or, otherwise, intensional concepts [24]:

- goals - a specific set of final and intermediate states, the achievement of which the agent has adopted as the current strategy of its behaviour;

- knowledge - a constant part of the agent's knowledge about itself, the environment and other agents, i.e. the part that does not change during its operation;

- beliefs - the agent's knowledge of the environment, in particular, about other agents; this is the knowledge that can change over time and become incorrect, but the agent may not have information about it and continue to remain in the belief that it is possible to base their conclusions on them;

- desires - states or situations, the achievement of which for various reasons is desirable for the agent, but they can be contradictory and therefore the agent does not expect that all of them will be achieved;

- intentions - what an agent is either obliged to do by virtue of his obligations to other agents (he is "assigned this task" and has taken on this), or what follows from his desires (i.e. a noncontradictory subset of desires, chosen for one reason or another, and which is compatible with the obligations assumed);

- commitments (obligations towards other agents) - tasks that the agent undertakes at the request (assignment) of other agents within the cooperative goals or goals of individual agents within the boundaries of cooperation.

In our architecture, agent-modules should be referred to as "strong" IAs due to their following mental properties.

The presence of goals for agents-modules is determined by strict regulation of the functions of the modules. Agentsmodules are required to have knowledge both about themselves and about other agents-modules (a constant part of the agent's knowledge about itself), since the system is able to solve the general task only as a whole, which none of the agent-module can independently accomplish. Only in the totality of interactions of full-featured agent-modules does the system as a whole achieve a given goal. The presence of this property in this case is also necessary because it is required to provide the "plug-and-play" mode in order to support the possibility of dynamic reconfiguration - rapidly replacement of the agent-module with a similar or modified one (which has a large number of mental properties). Moreover, during the reconfiguration process, each newly connected agent-module must inform all other agent-modules not only about its connection to the system, but also about its mental properties, as well as gain knowledge about other agent-modules. So, for example, when replacing conventional wheels in the transport module with omnidirectional wheels or "legs" (locomotors), the content of the TM mental properties is changed (the motion control algorithm is changed significantly), but the goal for the transport agent-module remains the same - to reach a given position in area. In this case, it is possible to change the interaction algorithm of agents-locomotors. Therefore, agents-locomotors should be provided with information interaction channels both with each other and with a higher level agent, which is a supervisor in relation to agents-locomotors.

The agent-module must have the benevolence property - the willingness of the agents to help each other to achieve a common goal and the agent's willingness to solve exactly those tasks assigned to him by the supervisor, which implies that the agent does not have conflicting goals. So, for example, when a TM moves on an uneven surface, each of the modules-locomotors at any time can have different conditions for movement, but for the transport module to execute a given velocity vector it needs to know the current state of other agents-locomotors so that they do not interfere each other.

Obviously, the module agents have the property of beliefs (knowledge about the environment, about other agents that may turn out to be wrong), since for a mobile robot, a priori knowledge about the environment and the state of other module agents can be invalid at any time. This knowledge may become incorrect, for example, if some sensors are damaged or even when individual agent modules fail, while the agent may not have information about it and continue to 
be convinced that everything is working fine. Beliefs should be based on previous experience (memory) and have a probabilistic character. To minimize errors, the system state should be constantly monitored, and the failure of any element of the system should be accompanied by a corresponding signal - "pain". Apparently, this property can be realized with the use of such mathematical apparatus as pentalogy [25].

Because of the implementation of agents-modules at the software and hardware level and their rigid functionality, such an agent property as mobility is possibly excluded, i.e. the agent's ability to migrate over the network in search of the necessary information to solve its tasks.

\section{Conclusion}

The considered functional-modular approach to the design of mobile robots control system, based on the hierarchical principle of distributed computing implementation, can be presented in the form of a specific multi-agent system. The modular architecture of mobile robot control system with such properties as full functionality, promptly reconfigurability and scalability, the ability to implement the "plug-and-play" mode and the modules feasibility on embedded computing devices can be considered as a multi-agent system.

In accordance with the agents classification according to the development degree of their internal understanding of the outside world and of the decision-making method, at each hierarchy level, hardware and software agents of different complexity can be used from a simple reflex agent (at the level of the transport module actuators - agent 4.1.1) to a learning agent (neural network at the level of the agent-ICM - agent 1.0). Conditions of use of agents of different complexity levels in this hierarchical multi-agent system will need to be determined in further research.

\section{Summary}

Proposed hierarchical topology (see Fig.3) has more similarities to a graph than a tree due to the presence of horizontal links between agents of the hierarchy same level (between agents 2.1, 2.2, 2.3; agents 3.1.1 and agents 3.3.1). The necessity of such interaction follows from the agents mental properties. However, when implementing this logical structure, a number of algorithmic difficulties may arise, and such interaction will have to be organized through a higher agent - agent-supervisor in relation to the considered group of agents. This, in turn, can cause an increase in the load on the appropriate communication channels, as well as require large computing power for the agent-supervisor. Future research in this direction will allow finding compromise solutions.

By using the theory of multi-agent systems, it will be necessary to find formal models of mental properties and rules of manipulation with them to describe the pyramidal structure of distributed computing in relation to the mobile robot control system. In addition, it is necessary to find a representation of the dynamic aspects of the functioning of both the individual agent-module and the community of agent-modules in mobile robots with a hierarchical modular architecture.

\section{Acknowledgments}

The Russian Foundation for basic research supports this research: Grant 19-07-00892a.

\section{References}

[1] Murata, S.; Yoshida, E.; Kamimura, A.; Kurokawa, H.; Tomita, K. \& Kokaji, S. (2002). M-TRAN: selfreconfigurable modular robotic system, IEEE/ASME Transactions on Mechatronics. - 2002. - No.7(4). - pp. 432-441.

[2] Østergaard, E.H.; Kassow, K.; Beck, R. \& Lund, H.H. (2006). Design of the ATRON lattice-based selfreconfigurable robot, Autonomous Robots. - 2006. - No.21(2). - pp.165-183.

[3] Guifang Qiao; Guangming Song; Jun Zhang; Hongtao Sun; Weiguo Wang \& Aiguo Song (2012). Design of Transmote: a Modular Self-Reconfigurable Robot with Versatile Transformation Capabilities, Proceedings of the 2012 IEEE International Conference on Robotics and Biomimetics, 2012. - pp.1331-1336.

[4] Ricardo Franco Mendoza Garcia; Andreas Lyder; David Johan Christensen \& Kasper Stoy (2009). Reusable Electronics and Adaptable Communication as Implemented in the Odin Modular Robot, IEEE International Conference on Robotics and Automation, 2009. - pp.1152-1158.

[5] Toshio Fukuda; Tsuyoshi Ueyama; Yoshio Kawauchi; Fumihito Arai (1987). Concept of cellular robotic system (CEBOT) and basic strategies for its realization, Computers Elect Engng. 1987. vol.18, no.1. - pp.11-39.

[6] Baca, J.; Ferre, M. \& Aracil R. (2012). A heterogeneous modular robotic design for fast response to a diversity of tasks, Robotics and Autonomous Systems. 2012. Vol.60. No.4. - pp.522-531.

[7] Lyder, A.H.; Stoy, K.; Mendoza-Garcia, R.F.; Larsen, J.C. \& Hermansen, P. (2013). On sub-modularization and morphological heterogeneity in modular robotics, Intelligent Autonomous Systems of Advances in Intelligent Systems and Computing. Springer Berlin Heidelberg. 2013. Vol.193. No.12. - pp.649-661. 
[8] Hancher, M.D. \& Hornby, G.S. (2006). A modular robotic system with applications to space exploration, 2nd IEEE International Conference on Space Mission Challenges for Information Technology (SMC-IT'06). Pasadena, CA: Publisher «IEEE». 2006. - pp.132-140.

[9] Mayoral, V.; Hernandez, A.; Kojcev, R.; Muguruza, I.; et al. (2017). The shift in the robotics paradigm - the Hardware Robot Operating System (H-ROS); an infrastructure to create interoperable robot components, NASA/ESA Conference on Adaptive Hardware and Systems (AHS), Pasadena, CA. - 2017. - pp.229-236.

[10] Herbrechtsmeier, S.; Korthals, T.; Schopping, T. \& Ruckert, U. (2016). AMiRo: a modular \& customizable opensource mini robot platform. 20th International Conference on System Theory, Control and Computing (ICSTCC), Sinaia. - 2016. - pp.687-692.

[11] Bonarini, A.; Matteucci, M.; Migliavacca, M .\& Rizzi, D. (2014). R2P: An open source hardware and software modular approach to robot prototyping. Robotics and Autonomous Systems. - 2014. - No.62. - pp.1073-1084.

[12] Losada, D.P.; Fernández, J.L.; Paz, E. \& Rafael Sanz (2017). Distributed and modular CAN-based architecture for hardware control and sensor data integration. Sensors. - 2017. - No.17. - pp.1013-1030.

[13] Andreev, V. \& Poduraev, Y. (2016). Network-Based Design of Heterogeneous Modular Mobile Robotic Systems, Proceedings of the 27th DAAAM International Symposium, pp.0004-0009, B. Katalinic (Ed.), Published by DAAAM International, ISBN 978-3-902734-08-2, ISSN 1726-9679, Vienna, Austria. DOI: 10.2507/27th.daaam.proceedings.001.

[14] Andreev, V.; Kim, V. \& Pletenev, P. (2017). The Principle of Full Functionality - the Basis for Rapid Reconfiguration in Heterogeneous Modular Mobile Robots, Proceedings of the 28th DAAAM International Symposium, pp.0023-0028, B. Katalinic (Ed.), Published by DAAAM International, ISBN 978-3-902734-11-2, ISSN 1726-9679, Vienna, Austria. DOI: 10.2507/28th.daaam.proceedings.003.

[15] Andreev, V. \& Kim, V. (2016). Control System and Design of the Motion Module of a Heterogeneous Modular Mobile Robot, Proceedings of the 27th DAAAM International Symposium, pp.0586-0594, B. Katalinic (Ed.), Published by DAAAM International, ISBN 978-3-902734-08-2, ISSN 1726-9679, Vienna, Austria. DOI: 10.2507/27th.daaam.proceedings.086.

[16] Andreev, V. \& Kim, V. (2017). The Application of Artificial Vector Fields for Motion Control of a Heterogeneous Modular Mobile Robot, Proceedings of the 28th DAAAM International Symposium, pp.0635-0644, B. Katalinic (Ed.), Published by DAAAM International, ISBN 978-3-902734-11-2, ISSN 1726-9679, Vienna, Austria. DOI: 10.2507/28th.daaam.proceedings.090.

[17] Andreev, V. \& Pletenev, P. (2017). Organizing Intermodular Communication for Heterogeneous Modular Mobile Robot, Proceedings of the 28th DAAAM International Symposium, pp.0474-0480, B. Katalinic (Ed.), Published by DAAAM International, ISBN 978-3-902734-11-2, ISSN 1726-9679, Vienna, Austria. DOI: 10.2507/28th.daaam.proceedings.066.

[18] Andreev, V.; Kim, V. \& Pletenev, P. (2018). Using Pareto Optimum to Choose Module's Computing Platforms of Mobile Robot with Modular Architecture, Proceedings of the 29th DAAAM International Symposium, pp.05590565, B. Katalinic (Ed.), Published by DAAAM International, ISBN 978-3-902734-20-4, ISSN 1726-9679, Vienna, Austria. DOI: 10.2507/29th.daaam.proceedings.081.

[19] Andreev, V. \& Tarasova, V. (2018). Identification of the Obstacle Shape Using the Ultrasonic Sensors Module of Modular Mobile Robot, Proceedings of the 29th DAAAM International Symposium, pp.1001-1009, B. Katalinic (Ed.), Published by DAAAM International, ISBN 978-3-902734-20-4, ISSN 1726-9679, Vienna, Austria. DOI: 10.2507/29th.daaam.proceedings.143.

[20] Andreev V.P., Kim V.L., Pletenev P.F. (2018). Hardware \& Software Solution for Rapid Reconfiguration of Heterogeneous Robots, Mekhatronika, Avtomatizatsiya, Upravlenie, 2018, vol.19, no.6, pp.387-395. DOI: 10.17587/mau.19.387-395.

[21] https://bookucheba.com/informatsionnyie-tehnologii_1237/7intellektualnyie-multiagentnyie-sistemyi-76106.html (Rus). Accessed on: 2019-10-16.

[22] https://www.intuit.ru/studies/courses/11068/1102/lecture/17391. NOU INTUIT. Multi-agent technologies (Rus). Accessed on: 2019-10-16.

[23] Wooldridge, M. \& Jennings N.R. (1994). Agent Theories, Architectures, and Languages: A Survey. In: Intelligent Agents. ECAI-94 Workshop on Agent Theories, Architecture and Languages. Amsterdam, The Netherlands, August 8-9, 1994, (Eds. M.J.Wooldridge and N.R.Jennings). Proceedings. Springer Verlag, 1994, pp.3-39.

[24] https://refdb.ru/look/2448008-pall.html. Gorodetsky V.I., Grushinsky M.S., Khabalov A.V. Multi-agent system (review) (Rus). Accessed on: 2019-10-16.

[25] Pryanichnikov, V.E.; Aryskin, A.A.; Eprikov, S.R.; Kirsanov, K.B.; Khelemendik, R.V.; Ksenzenko, A.Ya.; Prysev, E.A. \& Travushkin, A.S. (2017). Technology of Multi-Agent Control for Industrial Automation with Logical Processing of Contradictions, Proceedings of the 28th DAAAM International Symposium, pp.1202-1207, B. Katalinic (Ed.), Published by DAAAM International, ISBN 978-3-902734-11-2, ISSN 1726-9679, Vienna, Austria. DOI:10.2507/28th.daaam.proceedings.167. 